\title{
ANALYSIS OF TWO-STAGE ENDO-ATMOSPHERIC SEPARATION USING STATISTICAL METHODS
}

\author{
Mohammad Samani, Seid H. Pourtakdoust \\ Department of Mechanical and Aerospace Engineering, Science and Research Branch, Islamic Azad University, \\ Tehran, Iran; e-mail:m.samani@srbiau.ac.ir
}

\begin{abstract}
In this paper, selection and analysis of an atmospheric two stage separation system is discussed. The main purpose of this system is to test a supersonic parachute projectile, where a stage separation occurs after the burn out. Subsequently, the parachute is ejected from the payload after a minimum elapsed time. The separation times, for the supersonic parachute ejection, as well as the time needed for a safe clearing distance between the two stages are two critical issues in the separation process. In this respect, the knowledge of the relative position between the two stages is necessary to assure a safe distance and in order to adjust the required system parameters. In addition, as the nature of the parameters involved in the separation process is not deterministic, it would be useful to utilize the concept of random variables in the dynamic modeling of the separation process. In this paper, the modeling and simulation of the separation process is initially performed and partially verified. Subsequently, an approximate statistical method is utilized to acquire some probabilistic information about the relative distances at the two critical times. According to the simulation results, the relative distance between the two stages falls in a safe region. Finally, Monte Carlo simulation is also performed for comparison and verification of the statistical results that indicated a small and acceptable deviation between the two approaches. Thus, it can be concluded that the simpler approximate statistical approach is also valid for uncertainty analysis and can provide valuable knowledge needed in the preliminary design phase of the separation system.
\end{abstract}

Keywords: separation, safe distance, uncertainty, statistical methods, Monte Carlo simulation

\section{Introduction}

If all performance and physical characteristics of the Endo-atmospheric separation and recovery system are exactly as predicted, the projectile will fly what is called the nominal trajectory. However in practice, there are always some deviations between the actual and the predicted values. These deviations are primarily due to errors in the manufacturing processes, measurement systems, atmospheric conditions as well as the parachute modeling. As a result, the projectile will usually not fly exactly on its nominal trajectory and thus, the safe distances will not be met. In this respect, the estimation of errors is important from the operational point of view. In addition, investigation of error sources and their effects on the system proper performance can help a designer of the separation system to optimize the structural parameters in order to guarantee the minimum safe distance between the two stages. The separation events are considered as critical parts of any mission. Any mechanical interference between the two separating bodies is likely to be catastrophic. The launch failures of Atlas Centaur in 1970 and Chinese Long March in 1992, improper injection of Titan's satellite in 1990, Pegasus in 1991 and Delta-2 in 1995 are some of the typical examples in which faulty separation systems are suspected to be the main culprits. Therefore, it is not surprising that the dynamics of separating stages has received considerable attention. Lochan et al. (1992) have analyzed separation dynamics of strap-on boosters from the core rocket utilizing the wind tunnel simulation data. Choi et al. (2002) 
developed an efficient three-dimensional aerodynamic-dynamical coupled code to simulate the separation dynamics of strap-on boosters in the dense atmosphere. Jeyakumar and Rao (2006) also developed a model for the dynamics of a satellite separation system. Cheng (1999) developed an analytical procedure based on a coupled gas/structure model to simulate the fairing separation events. Roshanian and Talebi (2008) utilized the Monte Carlo method for statistical analysis of a multistage launch vehicle. Saghafi and Khalilidelshad (2005) have performed a Monte Carlo based dispersion analysis on a typical rocket. Lochan and Adimurthy (1997) also analyzed the separation dynamics in the atmosphere. Saxena (1979) designed the upper stage jet impingement on a separated booster. Zhao et al. (2011) modeled and simulated dynamical characteristics of a launch vehicle and spacecraft connected by the clamp band.

In this study, a shortcut statistical method as well as the Monte Carlo (MC) technique are utilized to investigate the safe distance between the two separating stages. For this purpose, a fifteen-degree-of-freedom simulation code (SepPak) is developed using the basic laws of motion and the rotating Earth. The modular software consists of different components needed for the modeling and simulation such as aerodynamics, mass properties, motion equations, atmosphere, gravity and wind models plus a model for aerodynamics of the parachute and its inflation time. Obviously, a combination of many design and system parameters is needed for design of the separation system. Subsequently, a shortcut statistical analysis is performed to determine the expected values and variances of the desired uncertain parameters. Additionally, Monte Carlo simulation is performed for validation of the results that accounts for any system related uncertainties such as the measured body rates, relative velocities and the parachute drag coefficient.

\section{Mission scenario}

Supersonic parachutes tests are normally performed for re-entry of payloads, particularly for space related missions. Experimental setups for the chutes are usually planned for a wind tunnel or aboard an airplane. However, a test projectile can also be employed for this purpose. In the latter case, a separate propulsion system will also be needed to boost the parachute release system to supersonic speeds. Due to limitations of cost and time, a typical existing rocket system (RS) is examined for this study. The RS is capable of accelerating the payload to a supersonic speed of $M=1.5$. Subsequently, SepPak is initially tuned and prepared for the required analysis of this paper. As mentioned before, the statistical analysis will be performed using two approaches. The Monte Carlo technique suggested by many researchers such as Lochan et al. (1994), Choi et al. (2002) and Jeyakumar and Rao (2006) is ideal, specially for nonlinear systems. It is numerically based on the provision of random data and numerous execution of the simulation code that is achieved by basically adding an extra outer loop around the SepPak. However, $\mathrm{MC}$ is computationally intensive and requires a large number of simulations for convergence, especially in complex non-linear systems such as the system at hand requiring a multitude of 3-D interpolations. This fact is also observed by other researchers in systems with a large number of random variables and in which the history of the statistical parameters does matter (Cheng, 1999; Roshanian and Talebi, 2008). However, due to its more accurate results, it is used for validation of statistical results determined from another approximate analytic technique detailed in Section 7 of this article. The approximate analytical technique gives the average and standard deviation of the major parameters, see Saghafi and Kalilidelshad (2005).

\section{The separation system}

The separation system and its key parameters are briefly presented in this Section. The clamp band and initial safe distance between the two stages are considered crucial issues in any separa- 
tion system (Lochan and Adimurthy, 1997). In the present case, the clamp band is pre-selected and a spring with the constant $k_{s p}=20000 \mathrm{~N} / \mathrm{m}$ and displacement of $\left|\Delta_{s p}\right|=0.10 \mathrm{~m}$ is considered to produce impulsive drag and compensate the remaining thrust (Jeyakumar and Biswas, 2005). To create a safe distance in minimum time, attitude motors could also be used. Since the separation process is not deterministic and there are uncertainties in the system parameter selection, it would be useful to utilize random variables in the dynamic modeling of the separation process (Lochan et al., 1994). Figure 1a shows the parachute and the payload as well as the forces acting on them. Figure $1 \mathrm{~b}$ shows a sketch of the separation system under study.

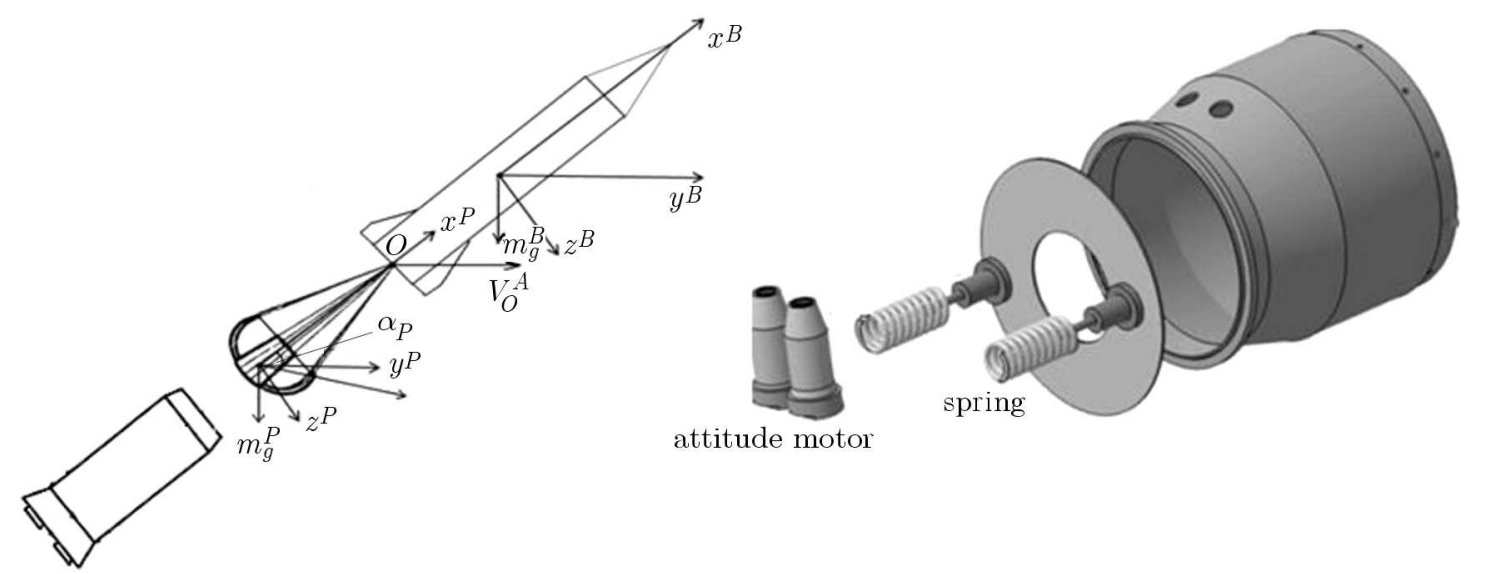

Fig. 1. (a) Parachute and payload; (b) separation system

\section{Dynamic modeling of separation}

In this Section, dynamic equations of motion of the separation are briefly discussed. Basic dynamic and kinematic equations of motion for the boosting as well as the separated stage are considered. The equations of motion of the first stage (booster) are similar to those of a usual flying vehicle, but the governing equations for the payload and the parachute are different and considered with more detail (Lochan et al., 1994; Lochan and Adimurthy, 1997; Jeyakumar and Biswas, 2005).

\section{Translational and rotational equations of motion}

According to Fig. 1, the translational and rotational equations of motion are (Singaravelu et al., 2013; Tewari, 2007)

$$
\begin{aligned}
& \sum_{k=1}^{2} \frac{d^{2} m^{B_{k}}}{d t^{2}} \mathbf{S}_{B_{k} I}=\sum_{k=1}^{2} \mathbf{f}_{k} \\
& m^{B} \mathbf{T}_{P B} \dot{\boldsymbol{\Omega}}_{E B}^{B} \mathbf{S}_{B O}^{B}+\left(m^{B}+m^{P}\right) \mathbf{V}_{E O}^{P}+\mathbf{T}_{P B} \boldsymbol{\Omega}_{B E}^{B} m^{B} \boldsymbol{\Omega}_{B E}^{B} \mathbf{S}_{B O}^{B} \\
& \quad+\boldsymbol{\Omega}_{P E}^{P}\left(m^{B}+m^{P}\right) \mathbf{V}_{E O}^{P}+m^{P} \dot{\boldsymbol{\Omega}}_{P E}^{P} \mathbf{S}_{P O}^{P}+\boldsymbol{\Omega}_{P E}^{P} m^{P} \boldsymbol{\Omega}_{P E}^{P} \mathbf{S}_{P O}^{P}=\sum \mathbf{f}^{P}
\end{aligned}
$$

where $m^{B}$ and $m^{P}$ are masses of the payload and the parachute, respectively, $\mathbf{T}_{P B}$ - transformation matrix of the parachute coordinates with respect to the payload coordinates, $\mathbf{V}_{E O}-$ velocity vector of the point $O$ in the rotating Earth coordinate system, $\boldsymbol{\Omega}_{B E}^{B}$-skew-symmetric matrix of the payload angular velocity vector $\boldsymbol{\omega}_{B E}, \boldsymbol{\Omega}_{P E}^{P}$ - skew-symmetric matrix of the payload angular velocity vector $\boldsymbol{\omega}_{P E}, \mathbf{S}_{B O}$ - payload dsiplacement vector, $\mathbf{S}_{P O}$ - parachute displacement vector, the vector $\mathbf{f}$ denotes the total aerodynamics and propulsion forces and $\mathbf{T}_{P B}$ represents the transformation matrix from the parachute to payload coordinate system. 
The aerodynamic $\mathbf{f}_{a}$ and the separation forces, $p$ are given as bellow (Jeyakumar and Biswas, 2003; Longren, 1970; Hurley and Carrie, 1972)

$$
\begin{aligned}
& \mathbf{f}_{a}=\left[\begin{array}{l}
f_{a_{x}} \\
f_{a_{y}} \\
f_{a_{z}}
\end{array}\right]=q^{\prime} S\left[\begin{array}{c}
-c_{A} \\
c_{Y} \\
-c_{N}
\end{array}\right] \quad q^{\prime}=\frac{1}{2} \rho\left|\mathbf{V}_{T}\right|^{2} \\
& p=k \Delta X=k\left(x_{0}-\left(x_{1}-x_{2}\right)\right)=k\left(x_{0}-\int_{0}^{t}\left(V_{1}-V_{2}\right) d t\right)
\end{aligned}
$$

where $p$ and $K$ are the separation force and the spring stiffness constant, respectively. $V_{1,2}=V_{1}-V_{2}$ and $X_{1,2}=x_{1}-x_{2}$ represent the relative velocity and relative distance between the stages. The general rotational equation of motion in an inertial coordinate system is given below (Tewari, 2007)

$$
\sum_{k=1}^{2} \frac{d}{d t} \mathbf{I} \boldsymbol{\omega}_{B_{k} I}=\sum_{k=1}^{2} \mathbf{M}_{O}
$$

where the left hand side can be expanded to give the following result for the system under study in parachute coordinate system using the terminology introduced in the nomenclature

$$
\begin{aligned}
\mathbf{T}_{P B} \mathbf{I}_{O B}^{B} \dot{\boldsymbol{\omega}}_{B E}^{B}+\mathbf{T}_{P B} \boldsymbol{\Omega}_{B E}^{B} \mathbf{I}_{O B}^{B} \boldsymbol{\omega}_{B E}^{B}+\mathbf{I}_{O P}^{P} \dot{\boldsymbol{\omega}}_{P E}^{P}+\boldsymbol{\Omega}_{P E}^{P} \mathbf{I}_{O P}^{P} \boldsymbol{\omega}_{P E}^{P} \\
\quad+\left(m^{B} \mathbf{T}_{P B} \mathbf{S}_{B O}^{B}+m^{P} \mathbf{S}_{P O}^{P}\right) \mathbf{V}_{I O}^{P}+\boldsymbol{\Omega}_{P I}^{P} m^{B} \mathbf{T}_{P B} \mathbf{S}_{B O}^{B} \mathbf{V}_{I O}^{B} \\
\quad+\boldsymbol{\Omega}_{P I}^{P} m^{P} \mathbf{S}_{P O}^{P} \mathbf{V}_{I O}^{P}=\sum \mathbf{M}_{O}^{P}
\end{aligned}
$$

where $\mathbf{I}_{O B}$ is the moment of inertia tensor matrix of the payload with respect to point $O$, $\mathbf{M}_{O}$ is the cumulative aerodynamic moment of the projectile, parachute and propulsion around the center of mass (Saxena, 1979; Singaravelu et al., 2013; Jeyakumar and Biswas, 2003). The aerodynamic moment about each axis is computed using its pertinent coefficient and the characteristic length $D$ represents the payload maximum body diameter

$$
\mathbf{M}_{a_{C G}}=\left[\begin{array}{l}
m_{a_{x}} \\
m_{a_{y}} \\
m_{a_{z}}
\end{array}\right]=q^{\prime} S D\left[\begin{array}{c}
c_{l} \\
c_{m} \\
c_{n}
\end{array}\right]
$$

The aerodynamic coefficients are derived using the MD software (Blake, 1997). The forces and moments of the parachute are also given below (Knacke, 1992)

$$
\left|\mathbf{f}_{P}\right|=\frac{1}{2} \rho\left|\mathbf{V}_{T}\right|^{2} S_{P} C_{D_{P}} \quad \mathbf{M}_{p}=\mathbf{r} \times \mathbf{f}_{p}
$$

where $S_{p}$ is the surface of parachute. The parachute drag coefficient $C_{D_{P}}$ is considered using the formulas given by Singaravelu et al. (2013) and Mohaghegh and Jahannama (2008)

$$
S_{p} C_{D_{P}}= \begin{cases}0.35 & \text { for } 5.5<t<8 \quad \text { (reefed) } \\ 0.65 & \text { for } t>8 \quad \text { (fully deployed) }\end{cases}
$$

To complete the set of equations, coupling of the two stages should also be considered. Thus,

$$
\mathbf{M}_{O}^{B}=\sum_{k=1}^{2} \frac{d}{d t} \mathbf{I}^{B} \boldsymbol{\omega}_{B_{k} I}^{B}-\mathbf{m}_{O}^{B}
$$

where $\mathbf{m}_{0}$ is the moment exerted by the payload around point $O$. 
Subsequently, the above equations can be joined together

$$
\begin{aligned}
& \mathbf{I}_{O B}^{B} \dot{\boldsymbol{\omega}}_{B E}^{B}+\boldsymbol{\Omega}_{B E}^{B} \mathbf{I}_{O B}^{B} \boldsymbol{\omega}_{B E}^{B}+\left(m^{B} \mathbf{S}_{B O}^{B} \mathbf{T}_{B P} \dot{\mathbf{V}}_{I O}^{P}\right) \\
& \quad+m^{B} \mathbf{T}_{B P} \boldsymbol{\Omega}_{P E}^{P} \mathbf{S}_{B O}^{B} \mathbf{T}_{B P} \mathbf{V}_{I O}^{P}=\sum\left(\mathbf{M}_{O}^{B}+\mathbf{m}_{O}^{B}\right)
\end{aligned}
$$

To derive the position of the two stages in the inertial frame, its corresponding linear and kinematic equations are considered. Thus,

$$
\left[\begin{array}{c}
\dot{x} \\
\dot{y} \\
-\dot{h}
\end{array}\right]=\mathbf{T}_{B I}\left[\begin{array}{c}
u \\
v \\
w
\end{array}\right] \quad \frac{d z}{d t}=-\frac{d h}{d t}
$$

where $\mathbf{T}_{B I}$ is the transformation matrix of the body coordinates with respect to the inertia coordinates. The required rotational kinematic equation for the transformation is based on the quaternions propagated as follows

$$
\dot{\mathbf{q}}=\frac{1}{2}\left[\begin{array}{cccc}
0 & -p & -q & -r \\
p & 0 & r & -q \\
q & -r & 0 & p \\
r & q & -p & 0
\end{array}\right] \mathbf{q} \quad \mathbf{q}=\left[\begin{array}{l}
q_{0} \\
q_{1} \\
q_{2} \\
q_{3}
\end{array}\right]
$$

The complete model of separation includes the aerodynamic forces, spring force, forces and moments of the parachute as well as the forces caused by the separation. By solving these equations, the position of each stage and relative distance between them will be determined

$$
d_{\text {relative }}=\sqrt{\left(x_{1}-x_{2}\right)^{2}+\left(y_{1}-y_{2}\right)^{2}+\left(h_{1}-h_{2}\right)^{2}}
$$

\section{Safe relative distance}

After the separation, the bag of the parachute is ejected through the gun system and subsequently the parachute bag, risers and cables are aligned along the axial direction. The length of the risers and suspension system is approximately $7.5 \mathrm{~m}$. Then the safe distance between the centers of masses of each stage, after ejection of the parachute bag, is selected as $15 \mathrm{~m}$. There always exist some uncertain parameters within the separation system, selection of which is difficult. The safe distance, in the parachute process, is one of them. Therefore, for the purpose of statistical analysis, it is considered as a random variable (Peggy, 2001; Bowker and Lieberman, 1972).

\section{Statistical analysis}

In general, since the design of the projectile separation system may involve several parameters to be considered, it would be useful to collectively keep these parameters in a random vector $\boldsymbol{\Phi}$. The random variable $\boldsymbol{\Phi}$, is assumed continuous and differentiable. Using Taylor series expansion (around the mean values) of the random variables $\bar{x}_{i}, i=1, \ldots, n$, and neglecting higher order terms, one can compute the expected value and variance of $\boldsymbol{\Phi}$ as follows

$$
E(\boldsymbol{\Phi}) \approx \boldsymbol{\Phi}\left(\bar{x}_{1}, \ldots, \bar{x}_{n}\right)+\sum_{i=1}^{n} \operatorname{var}\left(x_{i}\right) \frac{\partial^{2} \boldsymbol{\Phi}}{2 \partial x_{i}^{2}} \quad \operatorname{var}(\boldsymbol{\Phi})=\sigma^{2}(\boldsymbol{\Phi}) \approx \sum_{i=1}^{n} \operatorname{var}\left(x_{i}\right)\left(\frac{\partial \boldsymbol{\Phi}}{\partial x_{i}}\right)^{2}
$$

Since the random variables are considered with normal distribution, $\boldsymbol{\Phi}$ would have normal distribution (Wilke, 1964; Mitchell and Palmer, 1966). If the random variables are set in their 
normal region, the corresponding values of $\boldsymbol{\Phi}$ with the probability of 0.997 will be in $E(\boldsymbol{\Phi}) \pm 3 \sigma$ region (Jeyakumar et al. and Biswas, 2003).

The uncertainty in the system parameters such as mass, center of mass, moments of inertia, aerodynamic coefficients is considered using manufacturing tolerances, ground tests and some reference data (Choi et al., 2002; Jeyakumar and Rao, 2006). Because of the irregular shape of the payload dome and the uncertainty in drag coefficients derived from the MD software, more accurate and efficient methods are needed. Using the Fluent software (Fluent Inc., 2003), the drag force is computed to be $D=14250 \mathrm{~N}$ and the velocity contours are shown in Fig. 2a. The CFD results show $20 \%$ error with respect to the data obtained from the MD software. Thus, the payload drag force will be considered with a $\pm 30 \%$ uncertainty tolerance. Table 1 and 2 present the uncertainty levels chosen for various key parameters in the separation analysis as well as some of the characteristics of the two stages. Table 3 and 4 contain corresponding boundary values in the region of $[-3 \sigma,+3 \sigma]$ for the position of each stage and relative position between them after the parachute ejection. Time variations of the minimum relative distance as well as the mean relative distance between the two stages are shown in Fig. 2b. The above results have been obtained using the approximate statistical method mentioned in Eq. (7.1).
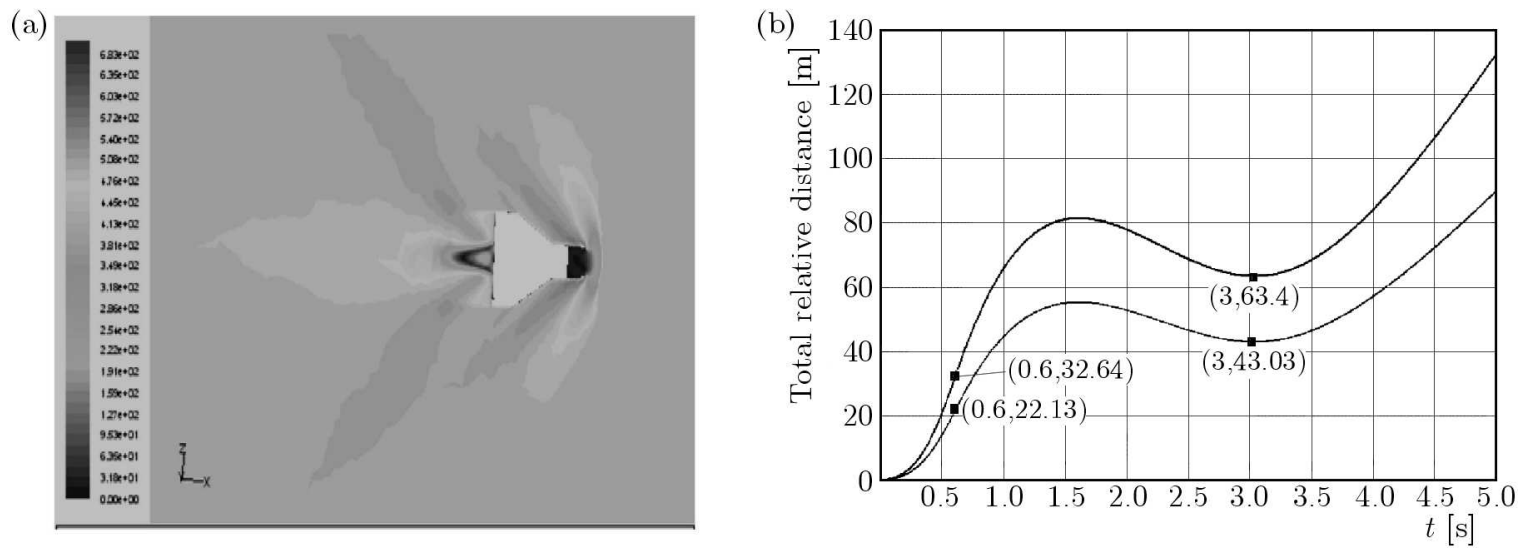

Fig. 2. (a) Stage 2 (payload). Velocity contours; (b) time variation of relative positions

Table 1. Sensitivity range for uncertain parameters (Roshanian and Talebi, 2008; Saghafi and Khalilidelshad, 2005)

\begin{tabular}{|l|c|}
\hline Parameter & Uncertainty ranges \\
\hline \hline residual thrust $[\mathrm{N}]$ & $\pm 1 \%$ \\
\hline$\left(C_{A}\right)_{\text {stage } 1}$ & $\pm 15 \%$ \\
\hline$\left(C_{A}\right)_{\text {stage } 2}$ & $\pm 30 \%$ \\
\hline$f_{\text {spring }}[\mathrm{N}]$ & $\pm 10 \%$ \\
\hline$\left(S C_{A}\right)_{\text {parachute }}$ & $\pm 30 \%$ \\
\hline
\end{tabular}

Table 2. Stage characteristics

\begin{tabular}{|l|c|c|c|c|c|}
\hline Parameter & $M[\mathrm{~kg}]$ & $x_{c g}[\mathrm{~m}]$ & $I_{x x}\left[\mathrm{~kg} \mathrm{~m}^{2}\right]$ & $I_{y y}\left[\mathrm{~kg} \mathrm{~m}^{2}\right]$ & $I_{z z}\left[\mathrm{~kg} \mathrm{~m}^{2}\right]$ \\
\hline \hline Stage 1 & 90 & 0.70 & 2.80 & 20 & 21 \\
\hline Stage 2 & 220 & 1.60 & 10 & 700 & 703 \\
\hline
\end{tabular}


Table 3. Statistical relative position of the two stages at the time of parachute inflation

\begin{tabular}{|c|c|c|c|}
\hline \multirow{2}{*}{ Parameter } & \multicolumn{3}{|c|}{ Statistical parameter } \\
\cline { 2 - 4 } & $-3 \sigma$ & Expected value & $+3 \sigma$ \\
\hline \hline$x[\mathrm{~m}]$ & 17.4 & 26.2 & 35 \\
\hline$y[\mathrm{~m}]$ & -1.2 & 2.50 & 6.3 \\
\hline$z[\mathrm{~m}]$ & 13.7 & 19.3 & 24.9 \\
\hline
\end{tabular}

Table 4. Relative distances at two critical times

\begin{tabular}{|c|c|c|c|c|}
\hline \multirow{2}{*}{ Parameter } & \multicolumn{4}{|c|}{ Statistical results } \\
\cline { 2 - 5 } & $t[\mathrm{~s}]$ & $E-3 \sigma$ & Expected value & $E+3 \sigma$ \\
\hline \hline$d_{\text {rel }}[\mathrm{m}]$ & 0.6 & 22.2 & 32.64 & 43.40 \\
\hline$d_{\text {rel }}[\mathrm{m}]$ & 3 & 43 & 63.4 & 83.8 \\
\hline
\end{tabular}

\section{Monte Carlo simulation}

The Monte Carlo technique is also utilized for stage separation analysis in this study. Monte Carlo simulation provides a unified framework for quantitative analysis of model uncertainty and assessment of the associated risk. It is also proven useful in the formulation of trade-off studies relative to design parameters. The use of high-speed computers makes application of the Monte Carlo simulation practical as a design and verification tool. Enhanced by the Monte Carlo technique, the separation analysis predicts the statistical bounds of separation parameters. These statistical bounds are used to assess the performance of the stage separation hardware design under the worst case conditions (Longren, 1970).

To validate the results of the statistical approach in this study, Monte Carlo simulation is also performed. For two sample time instances $(t=0.6 \mathrm{~s}$ and $t=3 \mathrm{~s})$, the expected value (running average) and the probability distribution functions of the relative distance between the two stages are calculated and presented in Figures 4-6. Figure 5 shows that the distribution of the payload drag coefficient to be normal and in the safe region, when the parachute is ejected and also during the approach phase. It is seen that the expected value (running average) of the relative distance between the two stages has converged to constant values in 20000 simulation loops of the Monte Carlo. The expected values as well as the $\pm 3 \sigma$ limits are given in Table 5 .
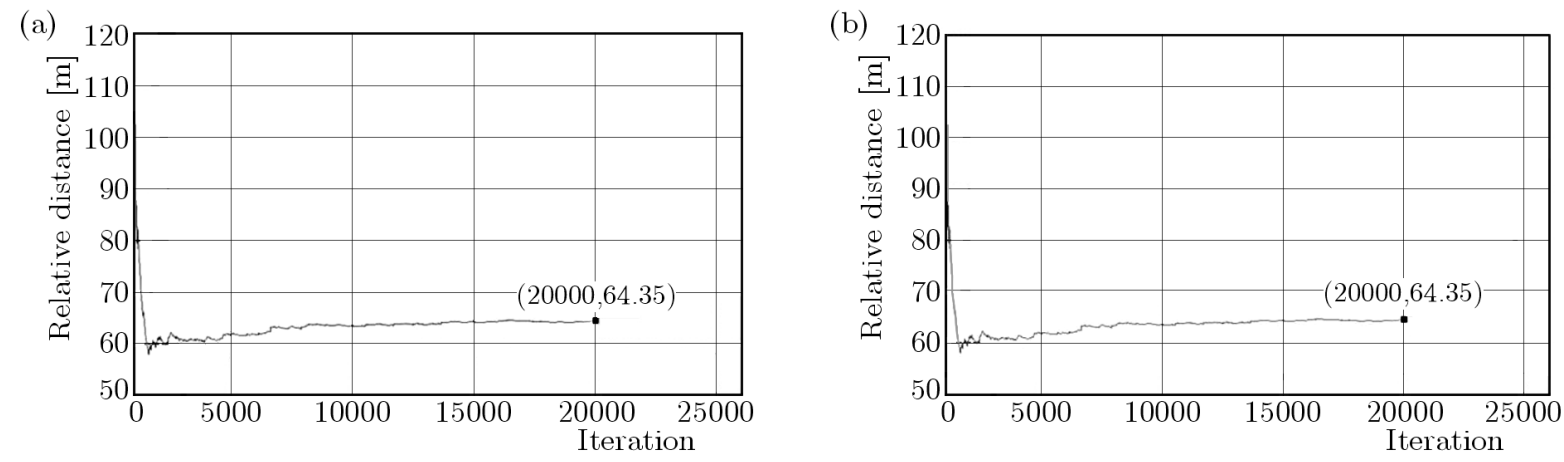

Fig. 3. Expected value of the relative distance at: (a) $t=0.6 \mathrm{~s}$, (b) $t=3 \mathrm{~s}$

\section{Conclusion}

In this paper, selection and analysis of a separation system for a supersonic two stage projectile is considered. An approximate statistical approach is initially utilized to determine the safe distance between two stages after parachute ejection. Although the attitude motors can accelerate the 

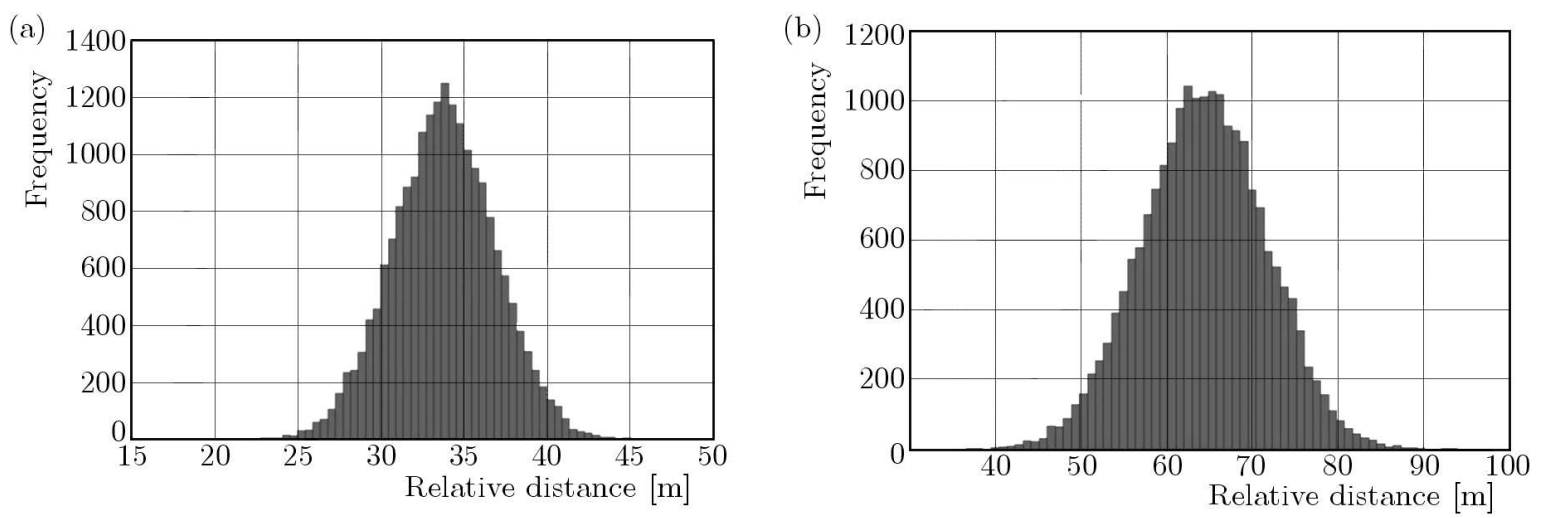

Fig. 4. Distribution of the relative distance at: (a) $t=0.6 \mathrm{~s}$, (b) $t=3 \mathrm{~s}$

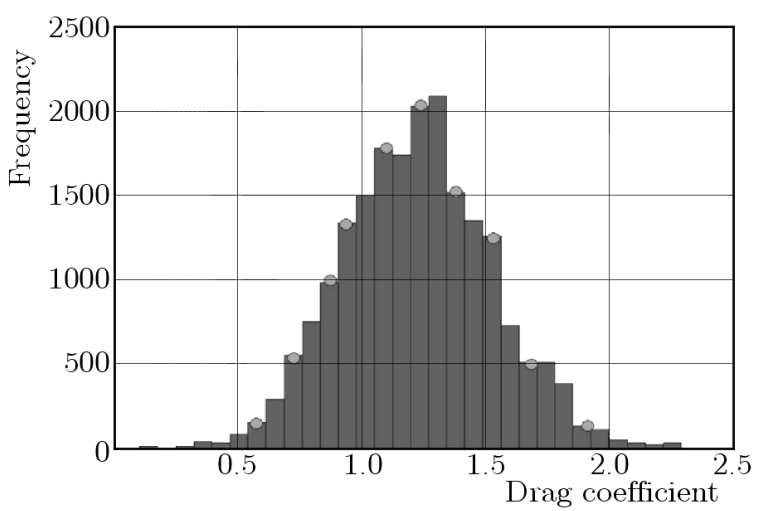

Fig. 5. Payload drag probability distribution function

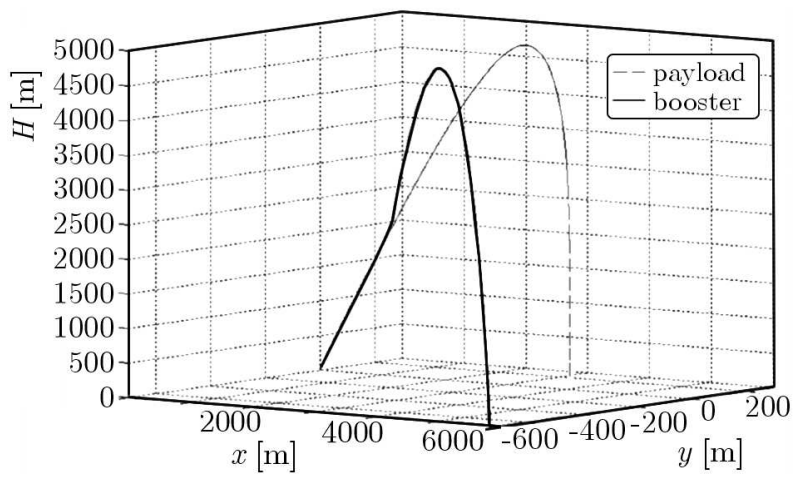

Fig. 6. Inertial position of the two stages

Table 5. Relative distances at critical times obtained from Monte Carlo analysis

\begin{tabular}{|c|c|c|c|c|}
\hline \multirow{2}{*}{ Parameter } & \multicolumn{4}{|c|}{ Statistical results } \\
\cline { 2 - 5 } & $t[\mathrm{~s}]$ & $E-3 \sigma$ & Expected value & $E+3 \sigma$ \\
\hline \hline$d[\mathrm{~m}]$ & 0.6 & 22.10 & 33.89 & 45.50 \\
\hline$d[\mathrm{~m}]$ & 3 & 43.50 & 64.40 & 85.30 \\
\hline
\end{tabular}


separation process, it is not needed for the present case. In order to validate the statistical results obtained from an approximate method, Monte Carlo analysis has been additionally performed. Based on the computed results, the expected values for both methods agree and differ only by $2 \%$ and $3.5 \%$, respectively for the relative safe distances at the two critical times. The standard deviations are also compatible and differ by $3 \%$ and $5 \%$, respectively for the same time instances. These values are acceptable and will be decreased by increasing the number of points in the normal distribution. Therefore, one can conclude that the approximate statistical approach is valid and can be used to obtain the required statistics about the relative position of the two separating stages.

\section{Reference}

1. Albertson C., Tartabini P., Pamadi B., 2012, End-to-end simulation of launch vehicle trajectories including stage separation dynamics, AIAA Atmospheric Flight Mechanics Conference

2. Blake B., 1997, Missile DATCOM User Manual, Ohio Air Force Research Laboratory

3. Bowker A.H., Lieberman G.I., 1972, Engineering Statistics, Prentice Hall Inc., Englewood, Cliffs, NJ

4. Cheng S.C., 1999, Payload fairing separation dynamics, Journal of Spacecraft and Rockets, 36, 4, $511-515$

5. Choi S., Kim C., Rho O.-H., Park J.-J., 2002, Numerical analysis on separation dynamics of strap-on boosters in the atmosphere, Journal of Spacecraft and Rockets, 39, 4, 439-446

6. Fluent Inc., 2003, Fluent User Manual.

7. Hurley M.J. Jr., Carrie G.W., 1972, Stage separation of parallel-staged shuttle vehicles: a capability assessment, Journal of Spacecraft and Rockets, 9, 10, 764-771

8. Jeyakumar D., Biswas K., 2003a, Design and analysis of the stage separation system of a massive liquid rocket stage, Proceedings of the International Conference on Modeling Simulation and Optimization for Design of Multidisciplinary Engineering Systems, Goa, India

9. JeYakumar D., Biswas K., 2003b, Stage separation system design and dynamic analysis of ISRO launch vehicles, Journal of Aerospace Sciences and Technologies, 55, 3, 211-222

10. Jeyakumar D., Biswas K., 2005, Stage separation dynamic analysis of upper stage of a multistage launch vehicle using retro rockets, Mathematical and Computer Modeling, 41, 8, 849-866

11. Jeyakumar D., Rao N., 2006, Dynamics of satellite separation system, Journal of Sound and Vibration, 297, 1, 444-455

12. Knacke T.W., 1992, Parachute Recovery Systems Design Manual, Parachute Recovery and Performances, 1st ed., Para Publishing, Santa Barbara, California

13. Lochan R., Adimurthy V., 1997, Separation dynamics of strap-on boosters in the atmosphere, Journal of Guidance, Control and Dynamics, 20, 4, 633-639

14. Lochan R., Adimurthy V., Kumar K., 1992, Separation dynamics of strap-on boosters, Journal of Guidance, Control and Dynamics, 15, 1, 137-143

15. Lochan R., Adimurthy V., Kumar K., 1994, Separation dynamics of ullage rockets, Journal of Guidance, Control and Dynamics, 17, 3, 426-434

16. Longren D.R., 1970, Stage separation dynamics of spin stabilized rockets, Journal of Spacecraft and Rockets, 7, 4, 434-439

17. Mitchell D.H., Palmer G.D., 1966, Analysis and simulation of a high accuracy spacecraft separation system, Journal of Spacecraft and Rockets, 3, 4, 458-463

18. Mohaghegh F., Jahannama M.R., 2008, Decisive role of filling time on classification of all parachute types, AIAA Journal of Aircraft, 45, 1, 267-275 
19. Papoulis A., 1991, Probability Random Variables and Stochastic Processes, McGraw-Hill

20. PegGy S.W., 2001, A Monte Carlo dispersion analysis of the X-33 simulation software, AIAA Atmospheric Flight Mechanics Conference, Montreal, Canada, 4067

21. Rao B.N., Jeyakumar D., Biswas K.K., Swaminathan S., Janardhana E., 2006, Rigid body separation dynamics for space launch vehicles, The Aeronautical Journal, 110, 1107, 289-302

22. Roshanian J., Talebi M., 2008, Monte Carlo simulation of stage separation dynamics of a multistage launch vehicle, Journal of Applied Mathematics and Mechanics, 29, 11, 1411-1426

23. Saghafi F., Khalilidelshad M., 2005, A Monte Carlo dispersion analysis of rocket simulation software, 6th Annual Conference of the Iranian Society of Aerospace Engineering, AERO2005

24. SAXENA S.K., 1979, Upper stage jet impingement on separated booster, Journal of the Royal Aeronautical Society, 616, 71-74

25. Singaravelu J., Sundaresan S., Nageswara Rao B., 2013, Application of fracture mechanics to specify the proof load factor for clamp band, systems of launch vehicles, Journal of Materials Engineering and Performance, 22, 4, 926-935

26. Tewari A., 2007, Atmospheric and Space Flight Dynamics, Birkhauser, Boston

27. Wilke R.O., 1964, Comments on coning effects caused by separation of spin stabilized stages, AIAA Journal, 2, 7

28. Wilkins M.P., 1999, Probability of collision error analysis, Space Debris, 1, 1, 21-35

29. Zhaо Y.-Q., YAn S.-Z., Chu F.-L., 2011, Dynamic characteristics of launch vehicle and space craft connected by clamp band, Journal of Sound and Vibration, 330, 10, 2161-2173

Manuscript received December 18, 2013; accepted for print July 10, 2014 